\title{
A review of microbial fuel cells coupled with constructed wetland
}

\author{
Zhenling $\mathrm{Li}^{1, \mathrm{a}}$, Chaowen Qian ${ }^{2, \mathrm{~b}}$, Yanli Ding ${ }^{3 *, \mathrm{c}}$, Shaoyuan Bai ${ }^{1,2, \mathrm{~d}}$, Xiaojun Lv ${ }^{1, \mathrm{e}}$ \\ ${ }^{1}$ Guangxi Key Laboratory of Environmental Pollution Control Theory and Technology, Guilin \\ University of Technology, Guilin 541004, China; \\ ${ }^{2}$ Collaborative Innovation Center for Water Pollution Control and Water Safety in Karst Area, Guilin \\ University of Technology, Guilin 541004, China; \\ ${ }^{3}$ College of Earth Sciences, Guilin University of Technology, Guilin 541004, China;

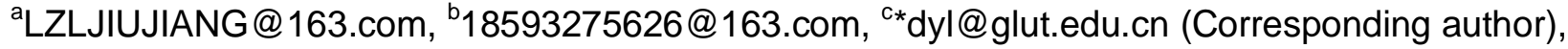 \\ dbaisy@glut.edu.cn, ${ }^{e} 1017888143 @ q q . c o m$
}

Keywords: microbial fuel cell, constructed wetland, influence factors

Abstract: Microbial fuel cell (MFC) coupled with constructed wetland (CW) is an innovative technology for wastewater treatment. Based on the current studies, the article reviewed the configuration and evaluation indicators of CW-MFC, and discussed the influence factors of MFC-CW performance, including $\mathrm{pH}$, electron donors and acceptors, electrode materials, the electrode spacing and $\mathrm{CW}$ matrixes. Key unresolved issues were concluded for potential applications of MFC-CW in the future.

\section{Introduction}

Constructed wetlands (CWs) are artificial systems that were designed and constructed to utilize the natural processes involving wetland vegetation, soils, and their associated microbial assemblages to purify wastewater[1]. They were often used for removing of organic matters and nutrients contained in wastewater[2]. For its low cost, coexistence with an inner aerobic/anaerobic environment, a high specific surface area of the matrix, a long hydraulic retention time (HRT) et.al.[3], constructed wetlands were regarded as a promising technology. However, with the running time increasing, constructed wetland suffers from a progressive media obstruction, so-called clogging process[4], causing the treatment performance degradation. It has become an urgent problem in recent years.

Nowadays, the study of microbial fuel cell (MFC) has been more and more popular. Progresses have been made in the MFC research and some review articles on the microbial fuel cell are available[5]. Compared with conventional wastewater treatment processes, MFC is an energy-harvesting platform incorporated into renewable energy stored in organic substances, particularly in wastewater, to produce bioelectricity and simultaneous wastewater treatment[6]. That is, it can convert chemical energy from organic matter into electrical energy by certain bacteria[7].According to previous study, Chemical energy that the wastewater contains is nine time more than the energy that is spent on purifying it in conventional wastewater treatment processes[8]. Thus, focusing on how to recycle and utilize this energy by the MFC has been more widely concerned[9].

However, MFCs is not isolated with the other wastewater treatment processes. MFCs were also more and more cooperated applications with other technologies, such as coupling with constructed wetland. Such coupling not only accelerate the wastewater treatment efficiencies, but also lower the costs and make the operation more easily ${ }^{[10]}$. For instance, Liam Doherty et al.(2015)[11] probed via operating a two-stage microbial fuel cell-constructed wetland concluded that a two-stage MFC-CW 
would increase the normalized energy recovery and improve removal efficiencies of COD, total nitrogen, $\mathrm{NH}_{4}{ }^{+}$, total phosphorus and reactive phosphorus to $93 \pm 1.7 \%, 85 \pm 5.2 \%, 90 \pm 5.4 \%$, $98 \pm 5.3 \%$ and $99 \pm 2.9 \%$, respectively. So, the microbial fuel cell coupled with constructed wetlands (MFC-CWs) is regarded as a promising technology.

\section{MFC-CWs Profile}

The structure and evaluation parameters of MFC-CWs. As a MFC, the MFC-CW consists of an anode compartment, a cathode compartment and an external circuit. The anode oxidizes organic compounds by electrochemically active microorganisms to release electrons and protons[11]. Then, electrons transfer to the cathode through the external circuit and generate electric currents. By measuring the produced current densities of MFC-CWs, wastewater treatment efficiencies are determined. It should be noted that electrochemically active microorganisms are anaerobic. However, oxygen usually acted as the electron acceptor in cathode, and the cathode must be aerobic. Additionally, the electrodes would be planted with aquatic plants in some MFC-CWs. Plants possess the unique ability to release oxygen as well as root exudates into the rhizosphere, and the excreted oxygen from plant roots could be used as a viable alternative source of electron acceptors for MFCs[12]. Liu et al. (2014)[13] pointed that wetland plant roots could be placed in the anode zone or cathode zone of an MFC, and they were both beneficial to power generation. Rhizosphere-anode CW-MFC is appropriate for treating low concentration organic wastewater, while the Rhizosphere-cathode CW-MFC has the ability of resisting high organic loading rate.

In general, recognized evaluation indicators of MFC-CWs are Coulomb efficiency (CE) and power density. $\mathrm{CE}$ is on behalf of the proportion of anodic organic compound which is loss of electrons. The higher of the electrons produced, the higher the $\mathrm{CE}$ is. The power density is the maximum power that per unit area electrode (anode or cathode) or per unit volume of liquor can output. It is positive relationship between the performance of MFC-CWs[3]. Comparing the performances among different structures of MFC-CWs depends on the size of the coulomb efficiency and power density.

Influence factors of MFC-CWs. There are many factors affecting the performance of the MFC-CWs, such as $\mathrm{pH}$, electron donors, electron acceptors, the electrode materials, electrode spacing, the matrix, influent factors, operation parameters and the presence of plants et al. For example, Zhou et al.(2013)[14] tested the performance of MFC-CWs for decolorization of azo dye and bioelectricity generation. The result indicated that the planted CW-MFC system achieved the highest decolorization rate of approximately $91.24 \%$ and a voltage output of approximately $610 \mathrm{mV}$. Concluding that plants grown in cathode compartment enhanced the cathode potential and slightly promoted dye decolorization efficiency. Besides Villaseñor, J. et al.(2013) ${ }^{[15]}$ conducted an experiment that the MFC-CW worked under continuous operation for $180 \mathrm{~d}$, treating three types of synthetic wastewater with increasing organic loading rates of $13.9 \mathrm{~g} \mathrm{COD} / \mathrm{m}^{2} \cdot \mathrm{d}, 31.1 \mathrm{gCOD} / \mathrm{m}^{2} \cdot \mathrm{d}$ and $61.1 \mathrm{gCOD} / \mathrm{m}^{2} \cdot \mathrm{d}$. The results indicated that under low organic loading rates, the wastewater organic matter was completely oxidized in the lower anaerobic compartment, and there were slight aerobic conditions in the upper cathodic compartment, thus causing an electrical current. However, under high organic loading rates, the organic matter could not be completely oxidized in the anodic compartment and flowed to the cathodic one, which entered into anaerobic conditions and caused the MFC-CW to stop working.

Oliveira et al. (2013)[5] pointed that $\mathrm{pH}$ had an effect on the MFC-CWs because the anode reaction released protons that will flow to the cathode compartment, which caused the increasing of 
$\mathrm{pH}$ in the cathode compartment. Current generation can significantly decrease according to the Nernst equation. Organic compounds which are oxidized decline affecting performance of MFC-CWs. Similarly, Zhang et al.(2016)[16] studied the effect of electrode material on the MFC. The findings from this study suggest that the $\mathrm{rGO} / \mathrm{MnO} 2 / \mathrm{CF}$ anode, fabricated via a simple dip-coating and electro-deposition process, could be a promising anode material for high-performance MFC applications.

Furthermore, Liam et al.(2015)[17] studied the effect of electrode spacing and flow direction on the performance of microbial fuel cell-constructed wetland. By placing the cathode at the air-water interface and burying the anode at a depth of $0.4 \mathrm{~m}$, the amount of dissolved oxygen at the cathode area is increased while ensuring the anode remains anoxic. He held that the ohmic resistance was reduced keeping the anode buried and placing the cathode directly above. Electrode spacing is attached a high importance to enhance performance of generate electricity, which is related to internal resistance. It is essential to enhance removal efficiency of pollutants via lowering the internal resistance.

\section{Prospects for MFC-CWs}

Nowadays, we seek energy sources that are high-effective, eco-friendly, and renewable to substitute the fossil fuels $[5,18,19]$, MFC-CWs is potential and will be more widely applied in the future. However, it is still a long way to go before the MFC-CWs are practical applied. There are a lot of problems in the need to be solved. Such as the clogging of MFC-CWs, Clara Corbella et al. (2016)[20]considered the microbial fuel cell coupled with horizontal subsurface flow constructed (HSSF CW) wetland to show a result that although longer study periods under more realistic conditions shall be further performed, the HSSF CW operated as a MFC has great potential for clogging assessment. In addition, especially, the recent studies indicated that the power density of MFC-CW is much lower than the traditional MFC for the smaller reactor volumes. The most reactor volumes of MFC-CW are still in the centimeter level, from $1.4 \mathrm{~L}$ to $96 \mathrm{~L}$ range[21]. However, the increased reactor volume might result in the decreasing of power density[21, 22]. For solving this problem, increasing the electrode ratio of the volume accounting, or the shrink electrode spacing, exploring multistage MFC-CW and operation mode are alternative methods.

\section{Acknowledgements}

This work was funded by the National Natural Science Foundation of China (No. 51408147, 41404116, 51638006), Science Research and Technology Development Project of Guangxi ( Guikehe1599005-2-2 )and was supported by the Guangxi Scientific Experiment Center of Mining, Metallurgy and Environment ( KH2012ZD004 ) and the project of high level innovation team and standing scholar in Guangxi colleges and universities ( 002401013001 )

\section{References:}

[1] J. Vymazal: Science of the Total Environment. 380. 1-3 (2007): p. 48-65.

[2] J. Vymazal: Ecological Engineering. 18. 5 (2002): p. 633-646. 
[3] S.H. Li Xianning, Xiang Wenli, Wu Lei: Journal of Southeast University(English Edition). 28. 02 (2012): p. 175-178.

[4] J. Nivala, P. Knowles, G. Dotro, et al.: Water Research. 46. 6 (2012): p. 1625-1640.

[5] V.B. Oliveira, M. Simões, L.F. Melo, et al.: Biochemical Engineering Journal. 73. (2013): p. 53-64.

[6] M.M. Mardanpour and S. Yaghmaei: Biosensors \& Bioelectronics. 79. (2016): p. 327-333.

[7] D. Pant, G. Van Bogaert, L. Diels, et al.: Bioresource Technology. 101. 6 (2010): p. 1533-1543.

[8] I. Shizas and D.M. Bagley: Journal of Energy Engineering-Asce. 130. 2 (2004): p. 45-53.

[9] S. Puig, M. Coma, J. Desloover, et al.: Environmental Science \& Technology. 46.4 (2012): p. $2309-2315$.

[10] K. Abe, M. Komada, A. Ookuma, et al.: Ecological Engineering. 69. (2014): p. 126-133.

[11] L. Doherty and Y. Zhao: Water Science and Technology. 72.3 (2015): p. 421-428.

[12] Z. Chen, Y.-c. Huang, J.-h. Liang, et al.: Bioresource Technology. 108. (2012): p. 55-59.

[13] S. Liu, H. Song, S. Wei, et al.: Bioresource Technology. 166. (2014): p. 575-583.

[14] Z. Fang, H.-L. Song, N. Cang, et al.: Bioresource Technology. 144. (2013): p. 165-171.

[15] J. Villaseñor, P. Capilla, M.A. Rodrigo, et al.: Water Research. 47.17 (2013): p. 6731-6738.

[16] C. Zhang, P. Liang, X. Yang, et al.: Biosensors \& Bioelectronics. 81. (2016): p. 32-38.

[17] L. Doherty, X. Zhao, Y. Zhao, et al.: Ecological Engineering. 79. (2015): p. 8-14.

[18] L.T. Angenent, K. Karim, M.H. Al-Dahhan, et al.: Trends in Biotechnology. 22.9 (2004): p. 477-485.

[19] J.H. Jo, D.S. Lee, D. Park, et al.: International Journal of Hydrogen Energy. 33. 19 (2008): p. 5176-5183.

[20] C. Corbella, J. García, and J. Puigagut: Science of The Total Environment.

[21] L.D. Wang Tong-yue, Zhao Xiao-hong,Zhao Ya-qian.: China water \& wastewater. 31.17 (2015): p. 8.

[22] Z. Ge, J. Li, L. Xiao, et al.: Environmental Science \& Technology Letters. 1. 2 (2014): p. 137-141. 